\title{
Osthole prevents acetaminophen-induced liver injury in mice
}

\author{
Yun CAI, Wu SUN, Xin-xin ZHANG, Yan-die LIN, Hao CHEN, Hao LI* \\ Key Laboratory of Cardiovascular Disease and Molecular Intervention, Department of Pathophysiology, Nanjing Medical University, \\ Nanjing 211166, China
}

\begin{abstract}
Acetaminophen (APAP) overdose leads to severe hepatotoxicity. Osthole, a natural coumarin found in traditional Chinese medicinal herbs, has therapeutic potential in the treatment of various diseases. In this study, we investigated the effects of osthole against APAP-induced hepatotoxicity in mice. Mice were administered osthole (100 $\mathrm{mg} \cdot \mathrm{kg}^{-1} \cdot \mathrm{d}^{-1}$, ip) for $3 \mathrm{~d}$, then on the fourth day APAP (300 $\mathrm{mg} / \mathrm{kg}$, ip) was co-administered with osthole. The mice were euthanized post-APAP, their serum and livers were collected for analysis. Pretreatment with osthole significantly attenuated APAP-induced hepatocyte necrosis and the increases in ALT and AST activities. Compared with the mice treated with APAP alone, osthole pretreatment significantly reduced serum MDA levels and hepatic $\mathrm{H}_{2} \mathrm{O}_{2}$ levels, and improved liver GSH levels and the GSSG-to-GSH ratio. Meanwhile, osthole pretreatment markedly alleviated the APAPinduced up-regulation of inflammatory cytokines in the livers, and inhibited the expression of hepatic cytochrome P450 enzymes, but it increased the expression of hepatic UDP-glucuronosyltransferases (UGTs) and sulfotransferases (SULTs). Furthermore, osthole pretreatment reversed APAP-induced reduction of hepatic cAMP levels, but pretreatment with H89, a potent selective PKA inhibitor, failed to abolish the beneficial effect of osthole, whereas pretreatment with L-buthionine sulfoximine, a GSH synthesis inhibitor, abrogated the protective effects of osthole on APAP-induced liver injury, and abolished osthole-caused alterations in APAP-metabolizing enzymes. In cultured murine primary hepatocytes and Raw264.7 cells, however, osthole (40 $\mu$ mol/L) did not alleviate APAP-induced cell death, but it significantly suppressed APAP-caused elevation of inflammatory cytokines. Collectively, we have demonstrated that osthole exerts a preventive effect against APAP-induced hepatotoxicity by inhibiting the metabolic activation of APAP and enhancing its clearance through an antioxidation mechanism.
\end{abstract}

Keywords: acetaminophen; hepatotoxicity; osthole; oxidative stress; inflammatory cytokines; UDP-glucuronosyltransferases; sulfotransferases; H89; L-buthionine sulfoximine

Acta Pharmacologica Sinica (2018) 39: 74-84; doi: 10.1038/aps.2017.129; published online 12 Oct 2017

\section{Introduction}

Acetaminophen (APAP), a commonly used anti-pyretic and analgesic agent, is safe at therapeutic doses; however, its overdose can cause acute liver injury. At therapeutic doses, the majority ( $>90 \%$ ) of APAP is metabolized by hepatic glucuronidation or sulfation to form non-toxic compounds, followed by renal and biliary excretion ${ }^{[1]}$. These reactions are catalyzed by UDP-glucuronosyltransferases (UGTs) and sulfotransferases (SULTs), respectively. Approximately $10 \%$ of APAP undergoes phase I oxidation by cytochrome P450 enzymes, mainly CYP2E1, to a toxic, highly reactive intermediate, N-acetyl-paminophenol (NAPQI) ${ }^{[1]}$. NAPQI is detoxified by conjugation to glutathione $(\mathrm{GSH})$ and excreted in the urine. However,

\footnotetext{
*To whom correspondence should be addressed.

E-mail haoli@njmu.edu.cn

Received 2017-03-09 Accepted 2017-06-05
}

APAP overdose saturates the glucuronidation and sulfation pathways, leading to increased generation and accumulation of NAPQI, which depletes cellular GSH, binds to cellular proteins, and promotes peroxynitrite production, thus generating excessive oxidative stress that leads to hepatocyte necrosis ${ }^{[2]}$.

Medical therapies for APAP-induced liver injury are limited. $\mathrm{N}$-acetyl cysteine (NAC), the only FDA-approved antidote, has partial efficacy, and it is most effective within 8-10 h after APAP overdose ${ }^{[3]}$. Side effects, such as nausea and vomiting, have been reported for NAC. Considerable efforts have been made to develop therapeutically effective agents from herbal medicines and natural products ${ }^{[4]}$.

Osthole (also known as osthol), 7-methoxy-8-(3-methyl-2butenyl)-2H-1-benzopyran-2-one, is a natural coumarin first derived from the Cnidium plant. High concentrations of osthole are found in the mature fruit of Cnidium monnieri (Fructus Cnidii), which is commonly used in the clinical practice 
of traditional Chinese medicine $(\mathrm{TCM})^{[5,6]}$. Osthole is also widely found in other medicinal plants including Angelica, Archangelica, Citrus, and Clausena. Osthole is demonstrated to have multiple functions, including anti-inflammation, antiosteoporosis, anti-tumor, anti-apoptosis, estrogen-like, hypoglycemic, anti-thrombosis and platelet aggregation effects ${ }^{[7]}$. The hepatoprotective effect of osthole has been widely investigated. Osthole has been reported to suppress the secretion of hepatitis B virus (HBV) in cell culture and prevent hepatitis induced by concanavalin A or anti-Fas antibody in mice ${ }^{[8-10]}$. Osthole has been proven to exhibit therapeutic effect on both hyperlipidemic ${ }^{[11]}$ and alcoholic fatty liver ${ }^{[12]}$ and to ameliorate hepatic fibrosis, inhibit hepatic stellate cell activation ${ }^{[13]}$, promote anti-tumor immune responses ${ }^{[14]}$, and inhibit growth of hepatocellular carcinoma ${ }^{[15]}$. Osthol was also shown to protect against liver damage in a rodent model of trauma-hemorrhage ${ }^{[16]}$. However, the effect of osthole on APAP-induced hepatotoxicity has not been assessed. In this study, we examined the effect of osthole on APAP-induced acute liver injury and investigated the underlying mechanism.

\section{Materials and methods}

\section{Animal husbandry and drug treatment}

Seven-week-old male BALB/C mice were used in this study. The mice were allowed free access to drinking water and food and were kept at room temperature $\left(25^{\circ} \mathrm{C}\right)$ on an automatic $12 \mathrm{~h}$ light and $12 \mathrm{~h}$ dark cycle. APAP (Sigma, USA) was dissolved in warm distilled water $\left(55-60{ }^{\circ} \mathrm{C}\right)$ and cooled to $37^{\circ} \mathrm{C}$, before it was intraperitoneally administered to mice. Osthole (purity $>98 \%$ ) was purchased from MedChem Express (Shanghai, China) and dissolved in a 1:9 $(v / v)$ mixture of Tween 80 and $0.9 \%$ sodium chloride. In most studies, mice were randomly assigned to four groups (control, APAP, osthole, APAP plus osthole). For the osthole and APAP plus osthole groups, osthole was intraperitoneally administered daily for $3 \mathrm{~d}$ at 100 $\mathrm{mg} / \mathrm{kg}$. On the fourth day, APAP $(300 \mathrm{mg} / \mathrm{kg})$ or its vehicle (osthole group) and osthole were co-administered to mice after they were fasted overnight. For the APAP group, osthole was replaced by its vehicle. The control group was treated with vehicle only. The mice were euthanized at specific time points after APAP injection. Their serum was collected, and their livers were harvested for further assays. In the dose-response study, osthole was administered at 15, 25, 50, $100 \mathrm{mg} / \mathrm{kg}$. In the indicated experiments, H89 (Sigma, USA, $10 \mathrm{mg} / \mathrm{kg}$ ), a specific PKA inhibitor, or L-buthionine sulfoximine (BSO, Sigma, USA, $1 \mathrm{~g} / \mathrm{kg}$ ), a glutathione depleting agent, was intraperitoneally administered $1 \mathrm{~h}$ prior to treatment with APAP. All animal protocols were pre-approved by the Animal Care and Use Committee of Nanjing Medical University.

\section{Serum chemistry analyses}

Enzymatic activities of serum alanine aminotransferase (ALT) and aspartate aminotransferase (AST) were spectrophotometrically estimated using commercially available kits (Jiancheng, China). Serum malondialdehyde (MDA) was measured using colorimetric assay according to the manufacturer's instruc- tions (Jiancheng, China).

\section{Determination of hepatic $\mathrm{H}_{2} \mathrm{O}_{2}$ and $\mathrm{GSH}$}

Frozen liver tissues were thawed and homogenized in icecold PBS. The homogenate was centrifuged at 3000 revolutions per minute at $4{ }^{\circ} \mathrm{C}$ for $10 \mathrm{~min}$, and the supernatants were assayed for $\mathrm{H}_{2} \mathrm{O}_{2}$ and GSH levels using commercially available assay kits according to manufacturer's instructions (Jiancheng, China).

\section{Measurement of cAMP and cGMP concentration}

The concentrations of cAMP, cGMP, CYP2E1, IL-1 $\beta$, and IL-6 were determined with commercial enzyme immunoassay kits (Elabscience, China). Frozen liver tissues were thawed and homogenized in ice-cold PBS. After centrifugation at 3000 revolutions per minute at $4{ }^{\circ} \mathrm{C}$ for $10 \mathrm{~min}$, the supernatants were analyzed for cAMP and cGMP levels according to the manufacturer's instructions.

\section{Western blotting}

Mouse liver samples were lysed with RIPA buffer containing protease inhibitors (Roche Diagnostics, Switzerland) for 15 min on ice. After centrifugation for $15 \mathrm{~min}$ at $20000 \times g\left(4^{\circ} \mathrm{C}\right)$, the protein content of the samples was determined using the Bradford method. Proteins (50 $\mu \mathrm{g}$ per lane) were separated using SDS-polyacrylamide gels and blotted onto methanolactivated PVDF membranes. The membranes were incubated with anti-mouse SULT2A1 (Elabscience, China) or anti-mouse UGT1A1 (Abcam, UK) antibodies at 1:1000. GAPDH from Sigma was used as internal control. Enhanced chemiluminescence with Amersham ECL Western blotting detection kit was performed according to the manufacturer's instructions (Amersham Biosciences, USA).

\section{Histopathology}

Liver tissues were fixed in $10 \%$ formalin and embedded in paraffin for histological assessment. Samples were subsequently sectioned $(5 \mu \mathrm{m})$, stained with hematoxylin and eosin (H\&E) and examined under a microscope (Olympus, Japan).

\section{Cell culture}

Primary hepatocytes were isolated from mouse liver by in situ collagenase perfusion through the portal vein. First, mice were anesthetized with pentobarbital sodium, and their livers were then perfused in situ with $45 \mathrm{~mL}$ liver perfusion medium, followed by $8 \mathrm{~mL}$ liver digestion medium. Then, the livers were excised, minced, and strained through a steel mesh. Hepatocytes were obtained by centrifugation 3 times at $50 \times g$ for 5 min each time and washed twice with Dulbecco's modified Eagle's medium (DMEM , Corning, USA). The hepatocytes were cultured in DMEM supplemented with 10\% FBS and penicillin/streptomycin for further experiments.

Raw264.7 cells were obtained from American Type Culture Collection (Manassas, VA, USA). The cells were maintained in DMEM supplemented with 10\% FBS and penicillin/streptomycin. 


\section{Cell viability assay using MTT}

The MTT assay was performed according to standard methods in 96-well plates. Briefly, $1 \times 10^{4}$ cells were seeded per well and cultured overnight before treatment with APAP and/ or osthole. At indicated time points, $10 \mu \mathrm{L}$ of $5 \mathrm{mg} / \mathrm{mL}$ MTT (Sigma) was added to each well, and after $4 \mathrm{~h}$ of incubation at $37^{\circ} \mathrm{C}$, the absorbance at $490 \mathrm{~nm}$ was measured with a microplate reader (SLT, Austria) according to the manufacturer's protocol.

\section{Quantitative real-time PCR}

Total cellular RNA was extracted using TRIzol reagent (Takara, Japan) according to the manufacturer's instructions. The total RNA ( $2 \mu \mathrm{g}$ ) was reverse transcribed using PrimeScript RT Reagent Kit (Takara, Japan). Real-time PCR was performed with Power SYBR Green PCR Master Mix (Applied Biosystems) using an Applied Biosystems 7500 Real-Time PCR System. The primer sequences are listed in Table 1. The relative quantities of mRNA were determined using the comparative cycle threshold method and normalized against GAPDH mRNA.

\section{Statistical analysis}

The data are expressed as the mean \pm SEM. Statistical significance was assessed by using unpaired Student's $t$-test. In all statistical comparisons, a $P$ value $<0.05$ was used to indicate a

Table 1. Primers used to detect gene expression at mRNA level.

\begin{tabular}{|c|c|}
\hline Gene name & Primer sequences ( $\left.5^{\prime}-3^{\prime}\right)$ \\
\hline \multirow[t]{2}{*}{ Nox2 } & F: GAAAACTCCTTGGGTCAGCACT \\
\hline & R: ATTTCGACACACTGGCAGCA \\
\hline \multirow[t]{2}{*}{ CYP3A11 } & F: ACCACCAGTAGCACACTTTC \\
\hline & R: CCAGGTATTCCATCTCCATCAC \\
\hline \multirow[t]{2}{*}{ CYP2E1 } & F: TGTGACTTTGGCCGACCTGTTC \\
\hline & R: CAACACACACGCGCTTTCCTGC \\
\hline \multirow[t]{2}{*}{ UGT1A1 } & F: CACCTGAAGCCTCAATACACAT \\
\hline & R: CAGTCCGTCCAAGTTCCACC \\
\hline \multirow[t]{2}{*}{ UGT1A6 } & F: GTTTCTCTTCCTAGTGCTTTGGG \\
\hline & R: CCTCGTTCACTGAGATGTTCTAC \\
\hline \multirow[t]{2}{*}{ UGT2B1 } & F: GTGCTGGTGTGGCCTACAG \\
\hline & R: CAGAAGATATGAGAACGGTGACG \\
\hline \multirow[t]{2}{*}{ SULT2A1 } & F: ССАТСТTСССАТССАТСТСТTС \\
\hline & R: GAGTGACCCTGGATTCTTCAC \\
\hline \multirow[t]{2}{*}{ IL-6 } & F: CTGCAAGAGACTTCCATCCAG \\
\hline & R: AGTGGTATAGACAGGTCTGTTGG \\
\hline \multirow[t]{2}{*}{ IL-1 $\beta$} & F: TTCAGGCAGGCAGTATCACTC \\
\hline & R: GAAGGTCCACGGGAAAGACAC \\
\hline \multirow[t]{2}{*}{ MCP-1 } & F: ACTGAAGCCAGCTCTCTCTTCCTC \\
\hline & R: TTCCTTCTTGGGGTCAGCACAGAC \\
\hline \multirow[t]{2}{*}{ CXCL10 } & F: CCAAGTGCTGCCGTCATTTTC \\
\hline & R: GGCTCGCAGGGATGATTTCAA \\
\hline \multirow[t]{2}{*}{ TNF $\alpha$} & F: CCAGGCGGTGCCTATGTCTC \\
\hline & R: CAGCCACTCCAGCTGCTCCT \\
\hline \multirow[t]{2}{*}{ GAPDH } & F: AGGTCGGTGTGAACGGATTTG \\
\hline & R: GGGGTCGTTGATGGCAACA \\
\hline
\end{tabular}

statistically significant difference.

\section{Results}

\section{Osthole attenuated APAP-induced liver injury}

Mice treated with $300 \mathrm{mg} / \mathrm{kg}$ APAP displayed severe liver injury at $6 \mathrm{~h}, 10 \mathrm{~h}$, and $12 \mathrm{~h}$ post-APAP, as indicated by increased serum ALT and AST activities (Figure 1A, 1B). Pretreatment with osthole $(100 \mathrm{mg} / \mathrm{kg})$ significantly attenuated the increase in ALT and AST activities. Similar alterations were detected in the activity of glutamate dehydrogenase (GLDH) (Supplementary Figure S1). Similar protective effects were also observed when osthole was administered simultaneously with APAP; however, when the mice were treated with osthole $0.5 \mathrm{~h}$ after APAP, no significant protection was observed (data not shown). Since pretreatment displayed more consistent results with different batches of osthole, we chose osthole pretreatment strategy for subsequent experiments. We found that the preventive effect of osthole against APAP-induced hepatotoxicity was dose dependent (Figure 1C, 1D). Even at the dose of $15 \mathrm{mg} / \mathrm{kg}$, the alleviation of APAP-induced liver injury by osthole was still significant. Consistently, histological assessment showed that osthole (100 $\mathrm{mg} / \mathrm{kg}$ ) reduced hepatocyte necrosis and intrahepatic hemorrhage induced by APAP (Figure 1E). These results indicated a significant beneficial effect of osthole against APAP-induced hepatotoxicity.

\section{Osthole inhibited APAP-induced oxidative stress in liver}

We detected various parameters of oxidative stress. The serum levels of MDA, which were dramatically increased with APAP overdose, were significantly reduced by osthole treatment (Figure 2A). Hepatic $\mathrm{H}_{2} \mathrm{O}_{2}$ levels were also significantly alleviated by osthole pretreatment when compared with APAP treatment alone (Figure 2B). The extent of oxidative stress was also assessed by GSH levels and the GSSG-to-GSH ratio (Figure 2C-2E). APAP resulted in a dramatic depletion of GSH and a marked increase of the GSSG-to-GSH ratio, while osthole significantly improved both parameters. As excessive accumulation of reactive oxygen species (ROS) occurs due to the imbalance between ROS generating and scavenging systems, we next compared the mRNA levels of several key genes involved in both processes using real-time PCR analysis. We found that osthole significantly suppressed the expression of pro-oxidant genes (Nox2, CYP3A11 and CYP2E1) (Figure $2 \mathrm{~F}$, Figure 3A-3C) without enhancing the expression of antioxidant genes (data not shown). Therefore, osthole inhibited APAP-induced oxidative stress mainly by reducing the formation of ROS.

\section{Osthole altered APAP-metabolizing enzymes}

To investigate the effect of osthole on APAP metabolism, using real-time PCR analysis, we first measured the expression of phase I enzymes that are known to facilitate the formation of toxic APAP metabolites. As indicated in Figure 3A, $3 \mathrm{~B}$, osthole significantly inhibited the increased expression of CYP3A11 and CYP2E1 genes in response to APAP. Expres- 

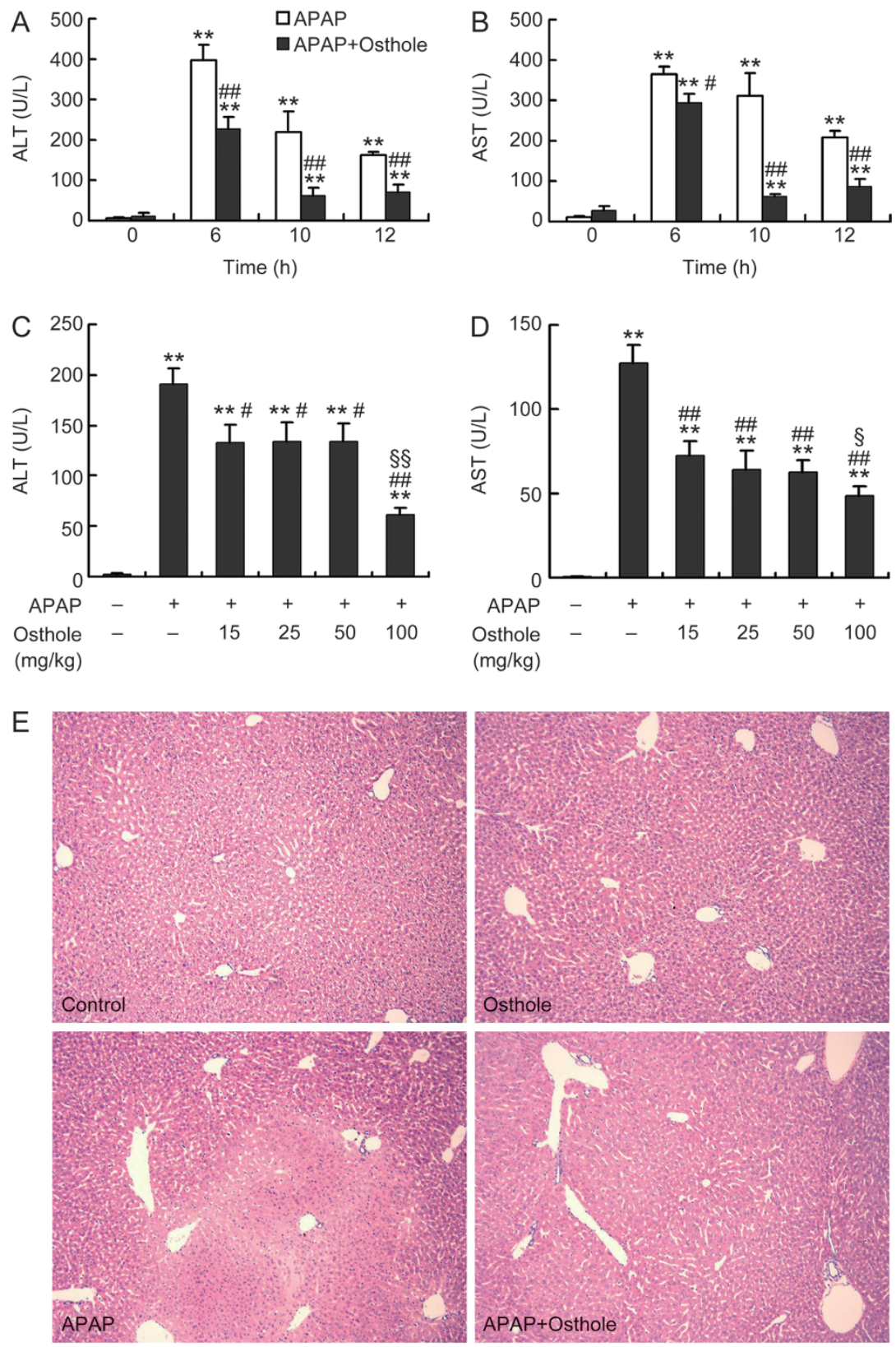

Figure 1. Osthole prevented APAP-induced liver injury. (A, B) Mice were intraperitoneally treated with osthole (100 mg/kg) for 3 consecutive days. On the fourth day, APAP $(300 \mathrm{mg} / \mathrm{kg}$ ) and osthole were simultaneously administered to mice. Mice were sacrificed at indicated time points post-APAP. Serum ALT and AST values were determined. (C, D) Dose-dependent responses of serum ALT and AST in mice in response to osthole at $6 \mathrm{~h}$ after APAP treatment. (E) Representative H\&E-stained liver sections from mice treated with APAP and/or osthole (100 mg/kg) (50×magnification). Data are expressed as the mean \pm SEM. $n=5-6$ mice per group. ${ }^{*} P<0.05,{ }^{* *} P<0.01$ compared to $0 \mathrm{~h}$ or vehicle. ${ }^{\#} P<0.05$, ${ }^{\#} P<0.01$ compared to APAP. ${ }^{\S} P<0.05$, ${ }^{\S \S} P<0.01$ compared to osthole at 15,25 , or $50 \mathrm{mg} / \mathrm{kg}$.

sion of hepatic CYP2E1 was also examined using ELISA. APAP induced a small $(0.05 \%)$ but significant increase in CYP2E1 protein in the liver, which was completely abolished by pretreatment with osthole (Figure 3C). Among the phase II enzymes, mRNA levels of UGT1A1, UGT1A6, UGT2B1, and SULT2A1 were increased in the osthole-pretreated mice compared with those in APAP only-treated mice (Figure 3D-3G). Similar alterations were also detected in protein lev- els of UGT1A1 and SULT2A1 by Western blotting (Figure 3H). These data demonstrated that osthole reduced APAP-induced hepatotoxicity by inhibiting its metabolic activation and promoting its clearance.

\section{Osthole reduced APAP-induced inflammatory response}

APAP-induced liver injury is associated with sterile inflammation, which is thought arise after the recruitment of 

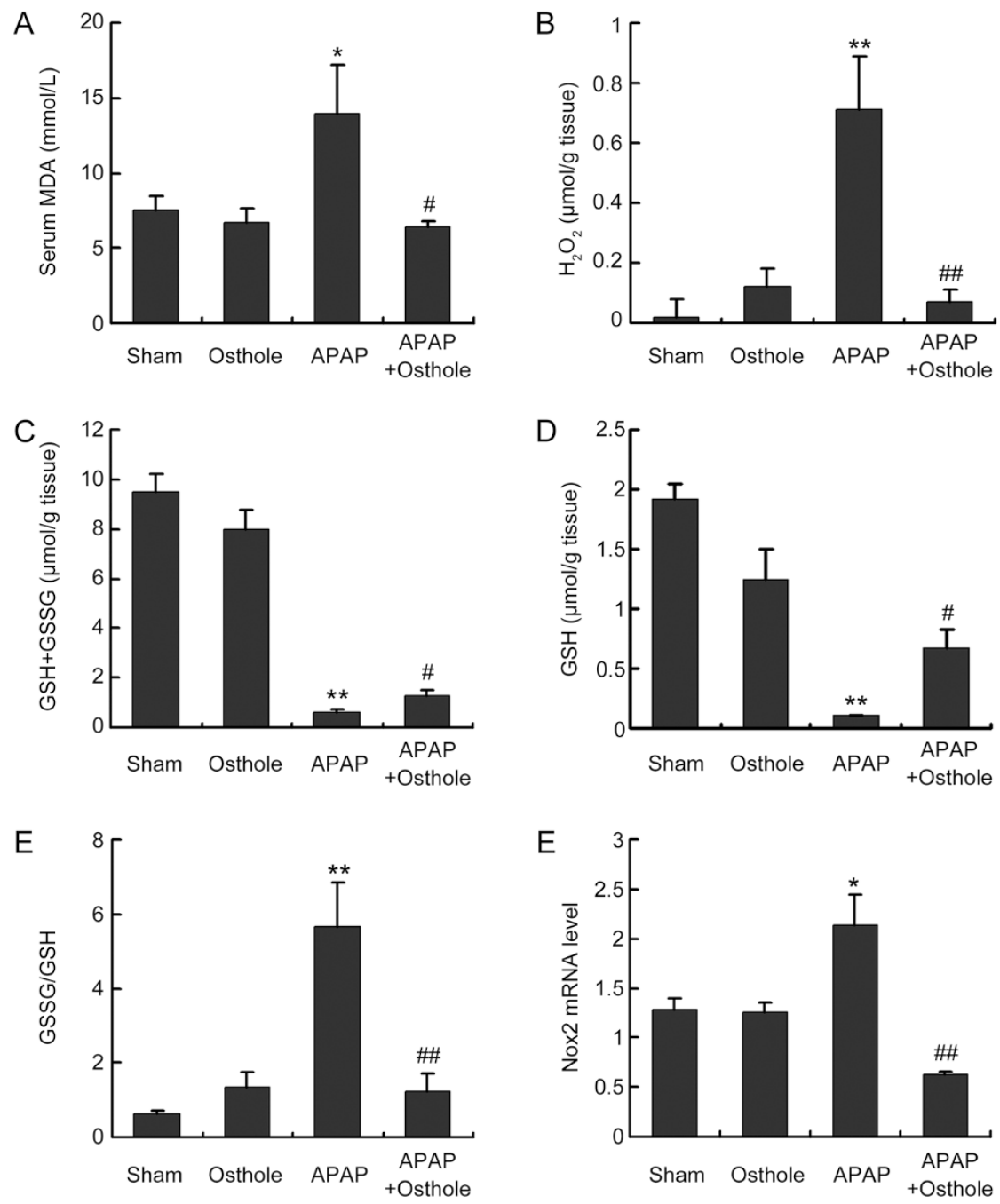

Figure 2. Osthole reduced APAP-induced oxidative stress. Mice were intraperitoneally treated with osthole (100 mg/kg) for 3 consecutive days. On the fourth day, APAP $(300 \mathrm{mg} / \mathrm{kg})$ and osthole were simultaneously administered to mice. Mice were sacrificed at $6 \mathrm{~h}$ post-APAP, and parameters of oxidative stress were determined. (A) Serum MDA; (B) Hepatic $\mathrm{H}_{2} \mathrm{O}_{2}$; (C) Hepatic GSH+GSSG; (D) Hepatic GSH; (E) GSSG-to-GSH ratio. (F) Relative mRNA levels of Nox2. Data are expressed as the mean \pm SEM. $n=5-6$ mice per group. ${ }^{*} P<0.05,{ }^{* *} P<0.01$ compared to control. ${ }^{\#} P<0.05$, ${ }^{\# \#} P<0.01$ compared to APAP.

inflammatory cells by damage associated molecular pattern molecules (DAMPs) ${ }^{[17]}$. To assess the effect of osthole on the generation of inflammatory cytokines, we first measured the expression of IL-1 $\beta$, IL- 6 and MCP-1 in mouse livers harvested at $6 \mathrm{~h}$ post-APAP using real-time PCR. The results showed that osthole pretreatment significantly reduced the expression of these cytokines compared with APAP treatment alone (Figure 4A-4C). Serum IL-1 $\beta$ and IL-6 levels detected by ELISA exhibited similar alterations (Figure $4 \mathrm{D}, 4 \mathrm{E})$. These findings were consistent with the known antiinflammatory activity of osthole.

Protective effect of osthole against APAP-induced hepatotoxicity was independent of the cAMP/PKA pathway

Accumulating studies have revealed that osthole causes a nonspecific elevation of intracellular and tissue cAMP and cGMP by inhibiting phosphodiesterases (PDEs), which can be attributed to the anti-inflammatory activity of osthole ${ }^{[18,19]}$. We therefore determined the cAMP and cGMP levels in mouse livers. Prominent reductions of cAMP $(P<0.05)$ and cGMP $(P=0.06)$ levels were observed at $6 \mathrm{~h}$ after APAP treatment. Osthole restored hepatic cAMP level to almost the level before APAP treatment but had no effect on cGMP levels (Figure 5A, 5B). cAMP mainly works by activating protein kinase A (PKA). Therefore, H89, a potent selective inhibitor of PKA, was used to test whether the hepatoprotective effect of osthole was mediated the cAMP/PKA pathway. The effect of H89 was confirmed by examining PKA activity in mouse livers (Supplementary Figure S2). We found that H89 pretreatment failed to abolish the osthole-induced reduction of ALT and AST activities (Figure 5C, 5D). Meanwhile, the osthole-induced alterations of genes involved in inflammation and metabolism of APAP were not reversed by H89 (Figure $5 \mathrm{E})$. These data indicated that the preventive effect of osthole 
A

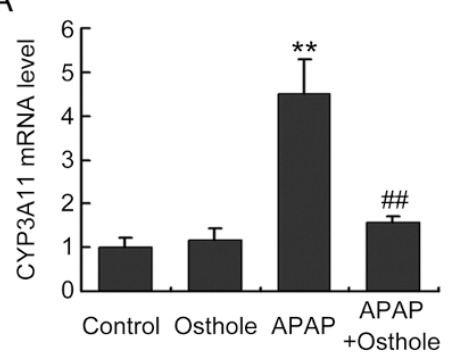

$\mathrm{D}$

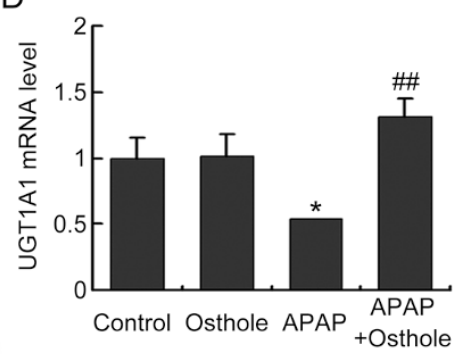

G

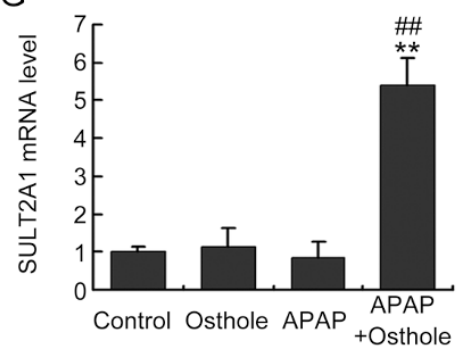

B

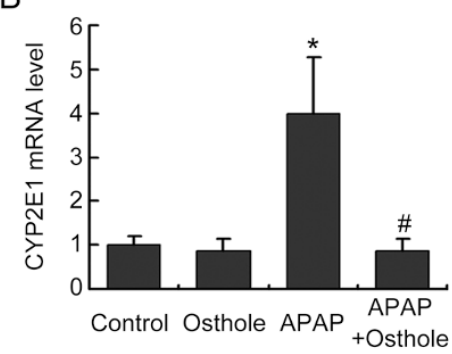

E

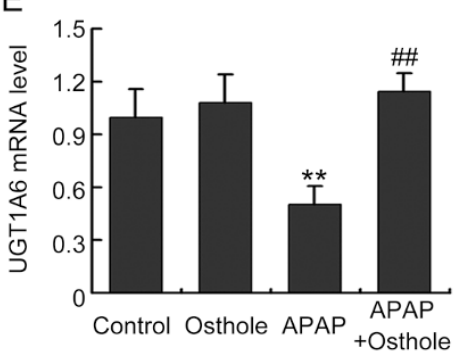

C

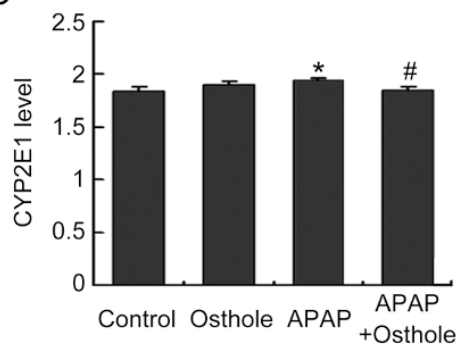

$\mathrm{F}$

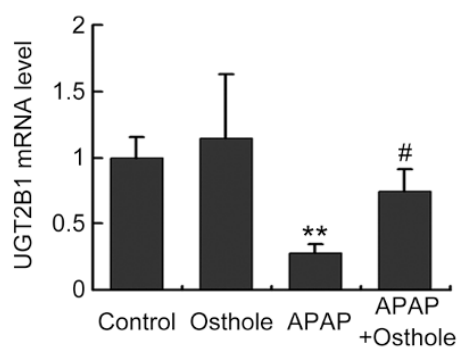

$\mathrm{H}$

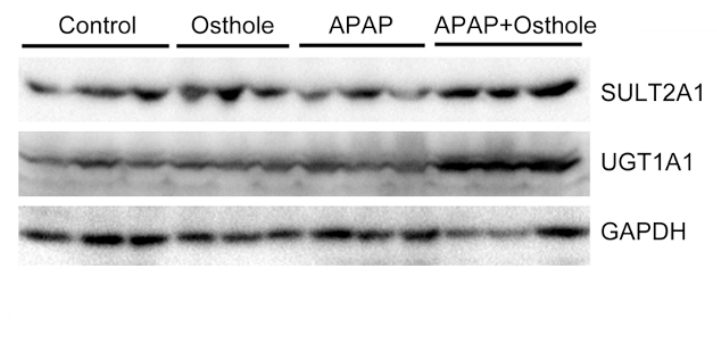

Figure 3. Osthole inhibited the metabolic activation of APAP and promoted APAP clearance. Mice were intraperitoneally treated with osthole (100 $\mathrm{mg} / \mathrm{kg})$ for 3 consecutive days. On the fourth day, APAP $(300 \mathrm{mg} / \mathrm{kg})$ and osthole were simultaneously administered to mice. Mice were sacrificed at $6 \mathrm{~h}$ post-APAP. (A, B) The mRNA levels of phase I enzymes in the liver were quantified using real-time PCR. (C) Hepatic CYP2E1 levels were determined using ELISA. (D-G) The mRNA levels of phase II enzymes in the liver were quantified using real-time PCR. (H) Hepatic SULT2A1 and UGT1A1 levels were determined by Western blotting. Data are expressed as the mean \pm SEM. $n=4-6$ mice per group. ${ }^{*} P<0.05,{ }^{* * *} P<0.01$ compared to control. ${ }^{\#} P<0.05$, ${ }^{\#} P<0.01$ compared to APAP.

against APAP-induced hepatotoxicity was not mediated by the cAMP/PKA pathway, and therefore this effect should not be attributed to the anti-inflammation property of this compound.

The hepatoprotective effect of osthole was mediated by its antioxidative property

Since osthole inhibited APAP-induced oxidative stress, we wanted to know whether antioxidation, another activity of osthole reported by numerous studies ${ }^{[20]}$, mediated its protective effect against APAP-induced liver injury. Determination of $E_{\text {lumo, }}$ a physicochemical parameter used in structure-activity relationship (SAR) analyses, suggested that the hepatoprotective activity of osthole may have been via its antioxidative properties ${ }^{[6]}$. To test this hypothesis, L-buthionine sulfoximine (BSO), an inhibitor of glutathione (GSH) synthesis, was intraperitoneally administered to mice $1 \mathrm{~h}$ prior to APAP exposure. The effect of BSO was confirmed by the evaluation of hepatic GSH levels (Supplementary Figure S3). We found that BSO pretreatment reversed the osthole-induced reduction of ALT and AST activities (Figure 6A, 6B). Real-time PCR analysis showed that BSO pretreatment led to a significant increase in MCP-1 but not of IL-1 $\beta$, compared with groups treated with osthole plus APAP (Figure 6C, 6D). Meanwhile, the ostholeinduced suppression of phase I enzymes CYP3A11 and CYP2E1 and induction of phase II enzymes UGT1A1, UGT1A6 and UGT2B1 were reversed by BSO pretreatment (Figure 6D). These results suggested that the hepatoprotective activity of osthole was likely mediated by its antioxidative properties.

\section{Osthole did not attenuate APAP-induced cell injury in vitro}

To assess the direct effect of osthole in vitro, freshly isolated primary mouse hepatocytes or Raw264.7 cells were treated with APAP, osthole or both as indicated (Figure 7A, 7B). Treatment with APAP alone caused extensive cell death indicated by MTT assay at $24 \mathrm{~h}$ post-treatment. Unexpectedly, pretreatment with osthole at 20,40, or $80 \mu \mathrm{mol} / \mathrm{L}$ did not alleviate APAP-induced cell death in either cell type. Real-time 

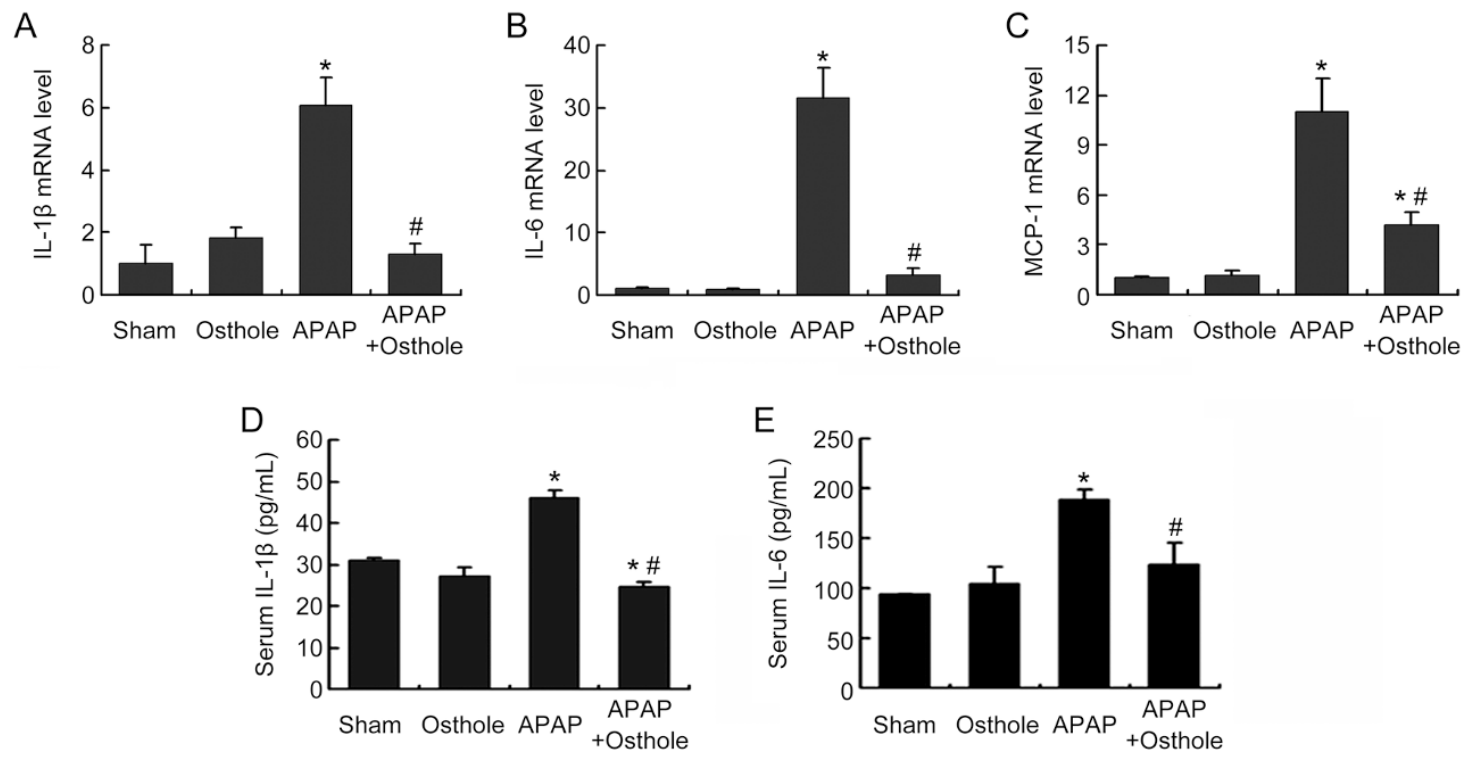

Figure 4. Osthole reduced APAP-induced inflammatory response. Mice were intraperitoneally treated with osthole (100 mg/kg) for 3 consecutive days. On the fourth day, APAP $(300 \mathrm{mg} / \mathrm{kg})$ and osthole were simultaneously administered to mice. Mice were sacrificed at $6 \mathrm{~h}$ post-APAP. (A-C) The mRNA levels of inflammatory cytokines in liver were quantified using real-time PCR. (D, E) Serum IL-1B and IL-6 levels were detected using ELISA. Data are expressed as the mean \pm SEM. $n=4-6$ mice per group. ${ }^{*} P<0.05$ compared to control and ${ }^{\#} P<0.05$ compared to APAP.

PCR analysis showed that osthole significantly suppressed the expression of inflammatory mediators such as CXCL10, IL-6, MCP-1, and TNFa (Figure 7C-7F); however, it failed to induce the phase II enzymes UGT1A1 and UGT1A6 or suppress the phase I enzymes CYP3A11 and CYP2E1 (Figure 7G-7J) in hepatocytes. These findings suggested that although osthole displays anti-inflammatory effects both in vivo and in vitro, it exhibits only antioxidative properties in vivo.

\section{Discussion}

Osthole, a natural product widely found in medicinal plants, especially Fructus Cnidii, has been demonstrated to display multiple pharmacological effects, including neuroprotective, osteogenic, immunomodulatory, anticancer, hepatoprotective, cardiovascular protective, and antimicrobial activities ${ }^{[20]}$. In this study, we showed that osthole exerts preventive effects against APAP-induced hepatotoxicity. Our findings enrich the current knowledge on the hepatoprotective activities of this coumarin.

It was revealed by SAR analyses that the presence of methylenedioxy/a hydroxyl group in the dibenzocyclooctadiene skeleton is an important structural feature for hepatoprotective activities ${ }^{[6]}$. Osthole contains 7-methoxy and 3-methyl2-butenyl groups in its structure, which are related to its hepatoprotective effect ${ }^{[21,22]}$. Since osthole demonstrated multiple pharmacological effects such as anti-inflammation, antioxidation, and immunomodulation, the goal of this study was to determine the preventive mechanisms of osthole against APAP-induced hepatotoxicity.

It is well established that APAP-induced hepatotoxicity is initiated by the formation of the reactive metabolite NAPQI, which can deplete liver GSH, modify cellular proteins, and promote excessive oxidative stress, which lead to hepatocyte necrosis ${ }^{[2]}$. The formation of NAPQI is primarily catalyzed by P450 enzymes, among which CYP3A4 has the highest activity, followed by CYP2E1 and CYP1A2, both at therapeutic and toxic concentrations ${ }^{[23]}$. As a homologous gene of CYP3A4, CYP3A11 plays a key role in metabolizing APAP into toxic NAPQI. On the other hand, the majority of APAP is either glucuronidated or sulfated and then excreted in the urine ${ }^{[1]}$. Therefore, the suppression of CYP2E1 and CYP3A11 and the induction of UGTs and SULT2A1 by osthole can account for its hepatoprotective effect.

$E_{\text {lumo }}$ values reflect electron affinities of respective compounds. $E_{\text {lumo }}$ values can be divided into three subsets corresponding to -1.12 to $-0.10,0.03$ to 1.07 , and 1.10 to $2.12^{[6]}$. These positive and negative ranges may indicate different mechanisms of action in hepatoprotection. $E_{\text {lumo }}$ values of osthole, coumarin, and flavonoids and their glucosides, ranging from -1.03 to -0.83 , suggest that their hepatoprotective activities may be achieved via their antioxidative properties, while glycyrrhizin, with its $E_{\text {lumo }}$ value ranging from 0.03 to 1.07, possibly exerts its hepatoprotective effects through other mechanisms (eg, anti-inflammation and/or immunomodulation $)^{[6]}$. In our study, although osthole exerted both antiinflammatory and antioxidative properties, BSO but not H89 impaired the preventive effect of osthole, indicating that this mechanism to protect against APAP-induced hepatotoxicity was antioxidation instead of anti-inflammation, which was consistent with the results of SAR analysis. We noted that BSO treatment affected the expression of phase I and II enzymes. In fact, it was demonstrated in many reports that the cellular redox status regulates the induction of UGTs ${ }^{[24]}$. Therefore, we speculate that the alterations in APAP-metabolizing enzymes 

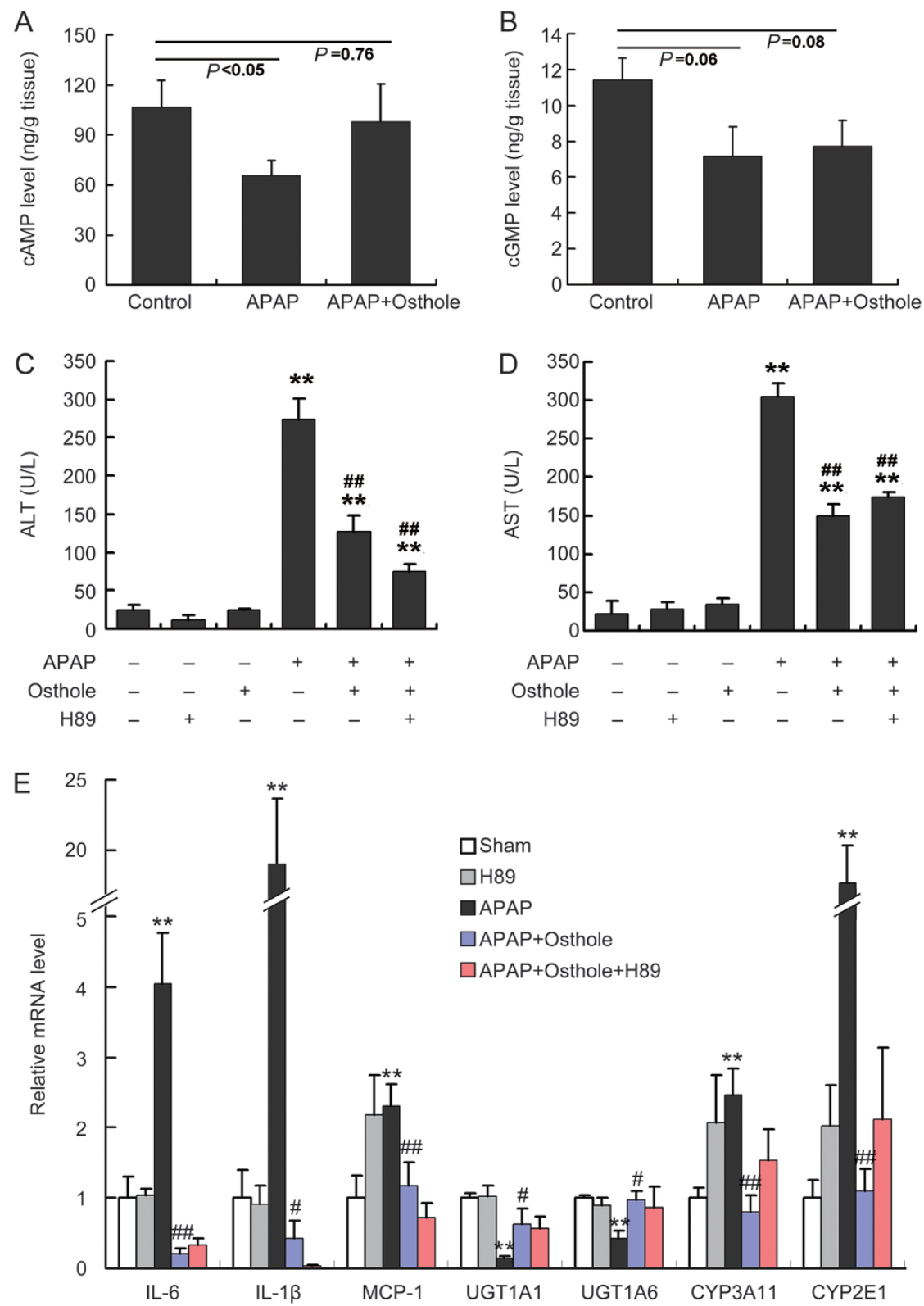

Figure 5. The preventive effect of osthole against APAP-induced hepatotoxicity was independent of the cAMP/PKA pathway. (A, B) Mice were intraperitoneally treated with osthole $(100 \mathrm{mg} / \mathrm{kg})$ for 3 consecutive days. On the fourth day, APAP $(300 \mathrm{mg} / \mathrm{kg})$ and osthole were simultaneously administered to mice. Mice were sacrificed at indicated time points post-APAP. Hepatic cAMP and cGMP levels were determined. (C, D) H89 (10 $\mathrm{mg} / \mathrm{kg}$ ) was intraperitoneally administered to mice $1 \mathrm{~h}$ prior to APAP treatment on the fourth day. Serum ALT and AST levels at $6 \mathrm{~h}$ post-APAP were determined. (E) The mRNA levels of genes involved in inflammation and metabolism of APAP in the liver were quantified using real-time PCR at $6 \mathrm{~h}$ postAPAP. Data are expressed as the mean \pm SEM. $n=4-6$ mice per group. ${ }^{* *} P<0.01$ compared to vehicle or control. ${ }^{\#} P<0.05$, ${ }^{\# \#} P<0.01$ compared to APAP.

induced by osthole were related to its antioxidative properties.

In APAP-induced hepatotoxicity, liver injury is associated with sterile inflammatory responses through the release of DAMPs from necrotic cells. However, there is extensive evidence against the role for neutrophils in the injury process after APAP overdose in mice or humans, although it cannot be excluded that some inflammatory mediators on intracellular signaling may have directly affected the injury process ${ }^{[2,25,26]}$. In our study, inhibition of the cAMP/PKA pathway, which accounts for the anti-inflammatory activity of osthole, failed to abolish the protective effect of osthole against APAP-induced hepatotoxicity. Consistently, osthole did not attenuate APAPinduced cell death in vitro despite its anti-inflammatory activity. These results suggested that the inhibition of oxidative stress but not inflammation is more important in the treatment of APAP-induced hepatotoxicity. In our study, MCP-1 and IL-1 $\beta$ responded differently to BSO, which may be related to the specific signaling pathways that regulate these cytokines. 

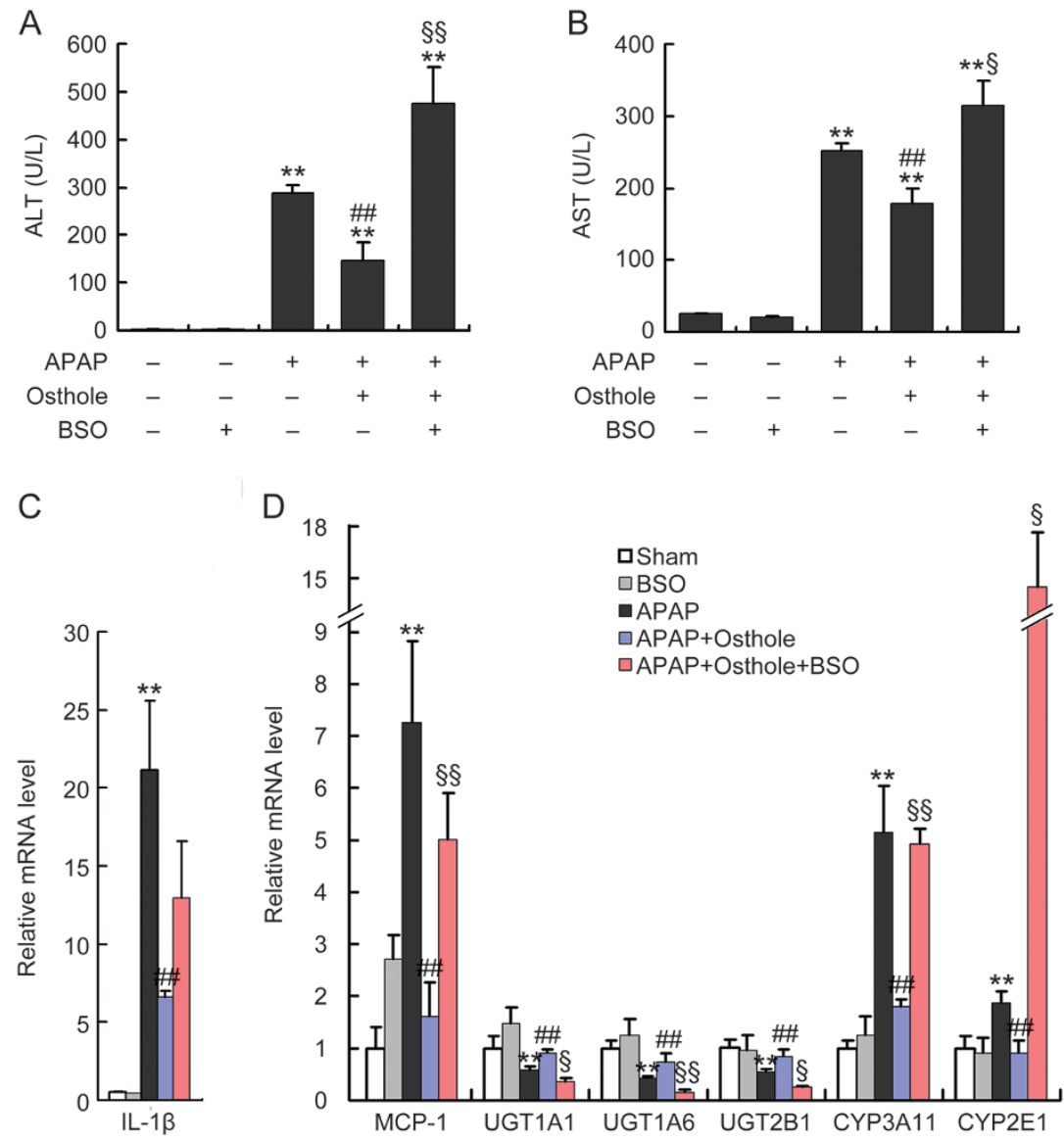

Figure 6. The hepatoprotective effect of osthole was mediated by its antioxidative property. (A, B) BSO $(1 \mathrm{~g} / \mathrm{kg})$ was intraperitoneally administered to mice $1 \mathrm{~h}$ prior to APAP treatment on the fourth day. Serum ALT and AST levels at $6 \mathrm{~h}$ post-APAP were determined. (C, D) The mRNA levels of genes involved in inflammation and metabolism of APAP in the liver were quantified using real-time PCR at $6 \mathrm{~h}$ post-APAP. Data are expressed as the mean \pm SEM. $n=4-7$ mice per group. ${ }^{* *} P<0.01$ compared to vehicle or control. ${ }^{\# \#} P<0.01$ compared to APAP. ${ }^{\S} P<0.05,{ }^{\S} P<0.01$ compared to APAP plus osthole.

We observed that osthole suppressed inflammation in isolated hepatocytes treated with APAP, which was consistent with previous studies suggesting the anti-inflammatory role of osthole in Raw264.7 cells ${ }^{[27]}$. We do not know the reason why osthole failed to protect cells against APAP-induced toxicity in vitro. One possibility might be that osthole is modified in vivo to become antioxidative or that some of its metabolites are antioxidants. Further studies are needed to clarify this matter.

In conclusion, we demonstrated that osthole exerts preventive effects on APAP-induced liver injury. This is mainly caused by the inhibition of metabolic activation of APAP and promotion of its clearance possibly through an antioxidation mechanism (Supplementary Figure S4). Osthole can be considered a potential natural resource for the development of hepatoprotective agents to prevent APAP overdose.

\section{Acknowledgements}

This study was supported by the National Natural Science Foundation of China (№ 81270944 and 81070678) and the Qinglan Project (2014) awarded to Dr Hao LI. Dr Hao LI is an associate fellow at the Collaborative Innovation Center for
Cardiovascular Disease Translational Medicine.

\section{Author contribution}

Yun CAI and Hao LI designed the study; Yun CAI, Wu SUN, Xin-xin ZHANG, and Hao CHEN performed the in vivo experiments; Yun CAI and Yan-die LIN were responsible for the in vitro experiments; Xin-xin ZHANG and Hao CHEN assisted in the care of the animals; Yun CAI, Yan-die LIN and Hao CHEN performed data analysis; Hao LI and Yun CAI wrote the manuscript.

\section{Supplementary information}

Supplementary information is available on the website of Acta Pharmacologica Sinica.

\section{References}

1 Bunchorntavakul C, Reddy KR. Acetaminophen-related hepatotoxicity. Clin Liver Dis 2013; 17: 587-607, viii.

2 James LP, McCullough SS, Knight TR, Jaeschke H, Hinson JA. Acetaminophen toxicity in mice lacking NADPH oxidase activity: role of peroxynitrite formation and mitochondrial oxidant stress. Free Radic 
A

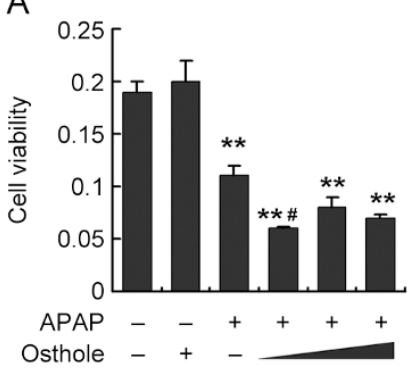

E

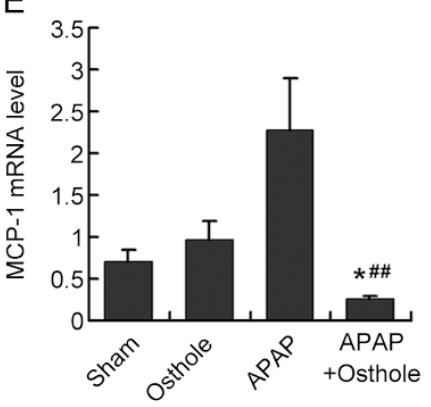

I

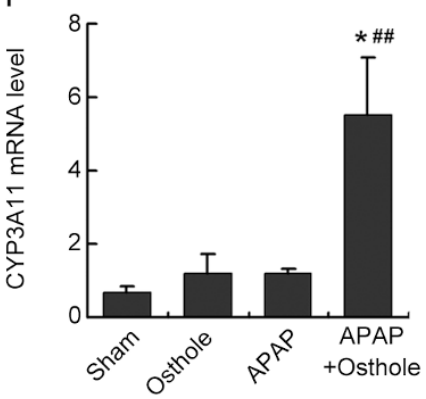

B

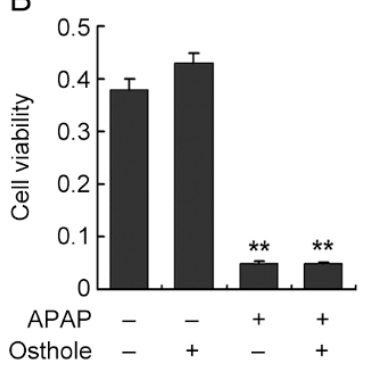

F

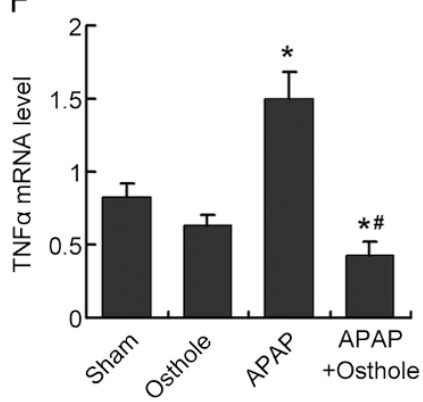

$\mathrm{J}$

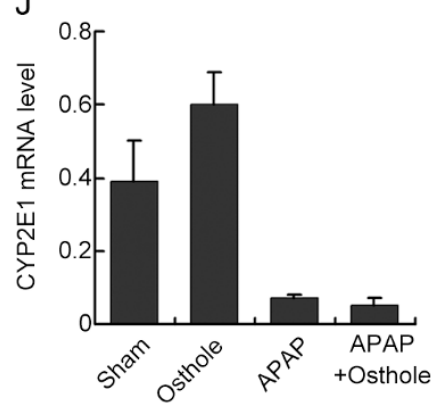

C

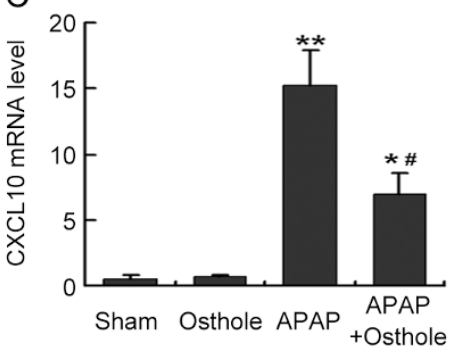

D

G

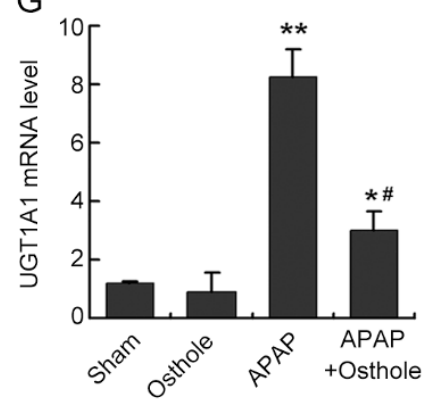

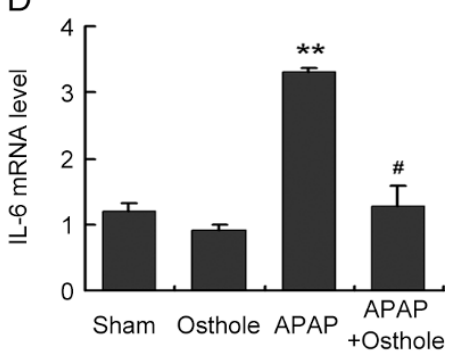

$\mathrm{H}$

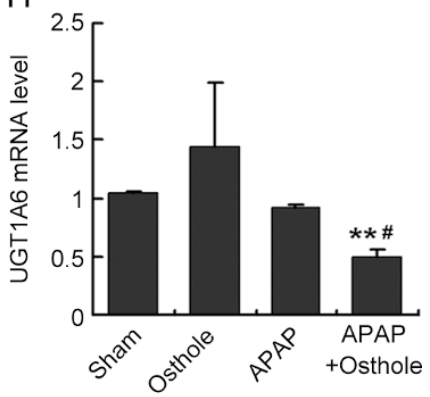

Figure 7. Osthole did not attenuate APAP-induced cell injury in vitro. (A) Primary mouse hepatocytes were isolated from mouse livers and incubated with $15 \mathrm{mmol} / \mathrm{L}$ APAP or $40 \mu \mathrm{mol} / \mathrm{L}$ osthole for $24 \mathrm{~h}$. Cell viability was determined using the MTT assay. To test the effect of osthole on APAP-induced cell death, primary hepatocytes were incubated with 20,40 , or $80 \mu \mathrm{mol} / \mathrm{L}$ of osthole for $24 \mathrm{~h}$ before the addition of APAP at $15 \mathrm{mmol} / \mathrm{L}$. Cell viability was determined at $24 \mathrm{~h}$ after treatment with APAP. (B) Raw267.4 cells were incubated with $40 \mu \mathrm{mol} / \mathrm{L}$ osthole for $24 \mathrm{~h}$ before the addition of APAP at $15 \mathrm{mmol} / \mathrm{L}$. Cell viability was determined using the MTT assay at $24 \mathrm{~h}$ post-APAP. The mRNA levels of genes involved in inflammation (C-F) and metabolism of APAP (G-J) in primary hepatocytes were quantified using real-time PCR at $24 \mathrm{~h}$ post-APAP. Data are expressed as the mean \pm SEM. $n=3$ wells per group. ${ }^{*} P<0.05,{ }^{* *} P<0.01$ compared to vehicle. ${ }^{\#} P<0.05,{ }^{\# \#} P<0.01$ compared to APAP.

Res 2003; 37: 1289-97.

3 Lee WM, Hynan LS, Rossaro L, Fontana RJ, Stravitz RT, Larson AM, et al. Intravenous $\mathrm{N}$-acetylcysteine improves transplant-free survival in early stage non-acetaminophen acute liver failure. Gastroenterology 2009; 137: 856-64.

4 Seeff LB, Lindsay KL, Bacon BR, Kresina TF, Hoofnagle JH. Complementary and alternative medicine in chronic liver disease. Hepatology 2001; 34: 595-603.

5 You L, Feng S, An R, Wang X. Osthole: a promising lead compound for drug discovery from a traditional Chinese medicine (TCM). Nat Prod Commun 2009; 4: 297-302.

6 Wang R, Kong J, Wang D, Lien LL, Lien EJ. A survey of Chinese herbal ingredients with liver protection activities. Chin Med 2007; 2: 5.

7 Hoult JR, Paya M. Pharmacological and biochemical actions of simple coumarins: natural products with therapeutic potential. Gen Pharmacol 1996; 27: 713-22.

8 Huang RL, Chen CC, Huang YL, Hsieh DJ, Hu CP, Chen CF, et al.
Osthole increases glycosylation of hepatitis B surface antigen and suppresses the secretion of hepatitis $B$ virus in vitro. Hepatology 1996; 24: 508-15.

9 Okamoto T, Kawasaki T, Hino 0. Osthole prevents anti-Fas antibodyinduced hepatitis in mice by affecting the caspase-3-mediated apoptotic pathway. Biochem Pharmacol 2003; 65: 677-81.

10 Okamoto T, Yoshida S, Kobayashi T, Okabe S. Inhibition of concanavalin A-induced mice hepatitis by coumarin derivatives. Jpn J Pharmacol 2001; 85: 95-7.

11 Qi Z, Xue J, Zhang Y, Wang H, Xie M. Osthole ameliorates insulin resistance by increment of adiponectin release in high-fat and highsucrose-induced fatty liver rats. Planta Med 2011; 77: 231-5.

12 Zhang J, Xue J, Wang H, Zhang Y, Xie M. Osthole improves alcoholinduced fatty liver in mice by reduction of hepatic oxidative stress. Phytother Res 2011; 25: 638-43.

13 Liu YW, Chiu YT, Fu SL, Huang YT. Osthole ameliorates hepatic fibrosis and inhibits hepatic stellate cell activation. J Biomed Sci 2015; 22: 
63.

14 Zhang L, Jiang G, Yao F, Liang G, Wang F, Xu H, et al. Osthole promotes anti-tumor immune responses in tumor-bearing mice with hepatocellular carcinoma. Immunopharmacol Immunotoxicol 2015; 37: 301-7.

15 Zhang L, Jiang G, Yao F, He Y, Liang G, Zhang Y, et al. Growth inhibition and apoptosis induced by osthole, a natural coumarin, in hepatocellular carcinoma. PLoS One 2012; 7: e37865.

16 Yu HP, Liu FC, Tsai YF, Hwang TL. Osthole attenuates hepatic injury in a rodent model of trauma-hemorrhage. PLoS One 2013; 8: e65916.

17 Jaeschke H, Williams CD, Ramachandran A, Bajt ML. Acetaminophen hepatotoxicity and repair: the role of sterile inflammation and innate immunity. Liver Int 2012; 32: 8-20.

18 Tsai YF, Yu HP, Chung PJ, Leu YL, Kuo LM, Chen CY, et al. Osthol attenuates neutrophilic oxidative stress and hemorrhagic shockinduced lung injury via inhibition of phosphodiesterase 4. Free Radic Biol Med 2015; 89: 387-400.

19 Liu J, Zhang W, Zhou L, Wang X, Lian Q. Anti-inflammatory effect and mechanism of osthole in rats. Zhong Yao Cai 2005; 28: 1002-6.

20 Zhang ZR, Leung WN, Cheung HY, Chan CW. Osthole: a review on its bioactivities, pharmacological properties, and potential as alternative medicine. Evid Based Complement Alternat Med 2015; 2015: 919616.
21 Okamoto T, Kajino K, Hino O. Hepatoprotective drugs for the treatment of virus-induced chronic hepatitis: from hypercarcinogenic state to hypocarcinogenic state. Jpn J Pharmacol 2001; 87: 177-80.

22 Okamoto T, Kobayashi T, Yoshida S. Chemical aspects of coumarin compounds for the prevention of hepatocellular carcinomas. Curr Med Chem Anticancer Agents 2005; 5: 47-51.

23 Laine JE, Auriola S, Pasanen M, Juvonen RO. Acetaminophen bioactivation by human cytochrome P450 enzymes and animal microsomes. Xenobiotica 2009; 39: 11-21.

24 Nguyen T, Sherratt PJ, Pickett CB. Regulatory mechanisms controlling gene expression mediated by the antioxidant response element. Annu Rev Pharmacol Toxicol 2003; 43: 233-60.

25 Cover C, Liu J, Farhood A, Malle E, Waalkes MP, Bajt ML, et al. Pathophysiological role of the acute inflammatory response during acetaminophen hepatotoxicity. Toxicol Appl Pharmacol 2006; 216: 98-107.

26 Williams CD, Bajt ML, Farhood A, Jaeschke H. Acetaminopheninduced hepatic neutrophil accumulation and inflammatory liver injury in CD18-deficient mice. Liver Int 2010; 30: 1280-92.

27 Liao PC, Chien SC, Ho CL, Wang El, Lee SC, Kuo YH, et al. Osthole regulates inflammatory mediator expression through modulating NFkappaB, mitogen-activated protein kinases, protein kinase $\mathrm{C}$, and reactive oxygen species. J Agric Food Chem 2010; 58: 10445-51. 